\title{
YY1-miR-146a-YY1 Regulatory Circuitry: A Potential Mechanism of Prostate Cancer Formation and Progression

\author{
M Chen ${ }^{1,2 *}$, N Tong ${ }^{3 *}$, S Chen ${ }^{1 *}$, Y Yang ${ }^{1}$, X Zhang ${ }^{1}$, H Jiang $^{1}$, N Liu ${ }^{1}$, C Sun ${ }^{1}$, J Liu ${ }^{1}$, G Sha ${ }^{1}$, W Zhu ${ }^{1}$, B Xu ${ }^{1,2}$
}

\section{ABSTRACT}

MicroRNA-146a (miR-146a) has been demonstrated as a tumour-suppressor in different malignancies including prostate cancer. As an endogenous small RNA, miR-146a regulates several gene expressions at the post-transcriptional level. Previous studies indicate that miR-146a is highly expressed in normal prostate tissue and significantly down-regulated in prostate cancer tissue and even worse in castrationresistant prostate cancer (CRPC) tissues. Over-expression of miR-146a in prostate cancer cell lines results in a marked reduction of cell proliferation, invasion and tumorigenesis. However, the regulating mechanism of miR-146a expression in the different stages of prostate cancer remains unclear. Yin Yang 1 (YY1), a critical regulator in prostate cancer development and progression, is predicted to be a direct target of miR-146a and binds to the promoter region of miR-146a, which is partly confirmed by the inverse expressions of miR-146a and YY1 in prostate cancer. Therefore, we hypothesize that YY1-miR$146 a-Y Y 1$ regulatory circuitry contributes to prostate cancer and may serve as a future intervention target.

Keywords: MicroRNA-146a (miR-146a), prostate cancer, regulatory circuitry, Yin Yang 1 (YY1)

WIMJ Open 2014; 1 (2): 42

\section{INTRODUCTION}

Prostate cancer is a common cause of cancer mortality and one of the most frequently diagnosed malignancies in males. In 2012, about 238590 new patients were diagnosed with prostate cancer and 29720 deaths were attributed to the disease in the United States of America [USA] (1). In the early stage of prostate cancer, patients are often sensitive to androgen ablation therapy; however, most often, androgendependent prostate cancer (ADPC) inevitably progresses to an androgen independent stage [castration-resistant prostate cancer (CRPC)] (2). Patients with CRPC often have a very poor prognosis since no curative treatment is currently available. It has been a major clinical challenge for doctors to reverse the transformation from ADPC to CRPC.

From: ${ }^{1}$ Department of Urology, Affiliated Zhongda Hospital of Southeast University, 87 Dingjia Bridge Hunan Road, Nanjing, 210009, China,

${ }^{2}$ Surgical Research Centre, Southeast University Medical School, 87 Dingjia Bridge Hunan Road, Nanjing, 210009, China and ${ }^{3}$ Department of Molecular and Genetic Toxicology, Cancer Centre of Nanjing Medical University, 140 Hanzhong Road, Nanjing 210029, China.

Correspondence: Dr B Xu, Department of Urology, Affiliated Zhongda Hospital of Southeast University, 87 Dingjia Bridge Hunan Road, Nanjing, 210009, China. E-mail: xb15896450810@126.com

${ }^{*}$ Contributed equally to the manuscript
MicroRNAs (miRNAs) are a class of small noncoding regulatory RNAs (about 19-25 nucleotides) which exert their function by binding to the 3'-untranslated region (3'-UTR) of a subset of mRNAs resulting in their degradation or repression of translation. Recent estimates suggest that one-third of human mRNAs may be regulated by miRNAs (3). During the progression of prostate cancer, several miRNAs and their targets have been discovered to express in an aberrant manner, which leads to the development, invasion and metastasis of this disease (4). Moreover, the aberrant expression of miRNAs has been found useful as biomarkers for diagnosis as well as prognosis of prostate cancer.

Many recent studies have suggested a role for miR146a in the development and maintenance of neoplastic processes as papillary thyroid carcinoma, pancreatic carcinoma, gastric cancer, breast cancer and prostate cancer. Lin et al (5) performed an miRNA array exhibiting a differential expression in CRPC as compared to ADPC cell lines, and found that miR-146a was one of the eight miRNAs repressed in CRPC cell lines. Further in situ hybridization analysis of miR-146a expression in prostate cancer tissues demonstrated that this miRNA is highly expressed in normal prostate tissue and significantly down-regulated in prostate cancer tissue and even worse in CRPC tissues. Over-expression of miR$146 \mathrm{a}$ in prostate cancer cell lines resulted in a marked reduc- 
tion of cell proliferation, invasion and metastasis to bone marrow through ROCK1. In our previous study (6), we found that miR-146a expression was significantly decreased in CRPC tissues compared to ADPC tissues. Functional analyses showed that ectopic over-expression of miR-146a in CRPC cell lines not only inhibited cell growth, colony formation, and migration in vitro, but also reduced tumorigenicity and angiogenesis in vivo. Mechanistic studies revealed that miR-146a repressed the expression of epidermal growth factor receptor (EGFR) through binding to the 3'-UTR in a dual phosphorylated extracellular signalregulated kinase (p-ERK) dependent manner. The consistent evidence showed that miR-146a might act as a tumour suppressor in prostate cancer progression from ADPC to CRPC through a complicated regulating network. However, the regulating mechanism of miR-146a expression in different stages of prostate cancer remains unclear.

\section{HYPOTHESIS}

The microRNA miR-146a impacts CRPC cell survival, thus it must be critically involved in CRPC formation and progression. Moreover, another important regulator in prostate cancer named Yin Yang 1 [YY1] $(7,8)$ is found not only as a direct target of miR-146a but also as a regulatory factor binding upstream of pri-miR-146a gene through bioinformatic analyses $(9,10)$. Based on these findings, we suppose that a YY1-miR-146a-YY1 regulatory circuitry may contribute to CRPC.

\section{EVIDENCE AND DISCUSSION}

MicroRNAs are a class of small, non-coding, single-stranded RNAs that negatively regulate gene expression by mainly binding to the 3'-UTR region of target mRNAs at the posttranscriptional level. MicroRNAs are required for many biologic processes, including cancer formation and progression. Recent work has demonstrated that miRNAs themselves were regulated by transcriptional factors directly binding to the sequence upstream pri-miRNA. For example, miR-221/222, two closely related miRNAs encoded in cluster from a genomic region on chromosome $\mathrm{X}$, were strongly upregulated in several forms of human tumours. It was identified that two separate distal regions upstream of miR-221/222 promoter were directly bound by the NF-kB subunit p65 and c-jun and both drove efficient transcription. Moreover, either the site-directed mutagenesis disrupting p65 binding sites or the ectopical inhibition of NF-kB activity significantly reduced transcriptional activity (11). Schiffgen and colleagues also found that the underlying mechanisms of microRNA deregulation in cancer cells include epigenetic modifications, which play a crucial role in carcinogenesis (12). They demonstrated that numerous miRNAs are induced in renal cell carcinoma cell lines after treatment with inhibitors of the DNA-methyltransferase (5-aza-2'-deoxycytidine) and the histone-deacetylase (suberoylanilide hydroxamic acid). Moreover, the enrichment of $\mathrm{H} 3$ and H3K18 acetylation at the miR-9 promoter led to re-expression of miRNA, while DNA hypermethylation remains unchanged (12).

Human miR-146a resides in the LOC285628 gene on human chromosome 5. The LOC285628 transcript contains no significant open reading frame (ORF), implying that it probably belongs to a class of noncoding RNAs. There are two-exon structures of the miR-146a primary transcript (primiR146a), and the mature miR-146a sequence situated in the second exon. We performed a bioinformatic search for transcription factor binding sites (TFSearch software, http:// www.cbrc.jp/research/db/TFSEARCH.html) in the human genomic sequence $(20 \mathrm{kbp})$ upstream of miR-146a transcriptional unit and identified predicted six YY1 binding sites embedded in two regions highly conserved in mammals, as evidenced from Human Mar. 2006 (NCBI36/hg18) assembly UCSC Genome Browser [http://genome.ucsc.edu/] (Fig.1).

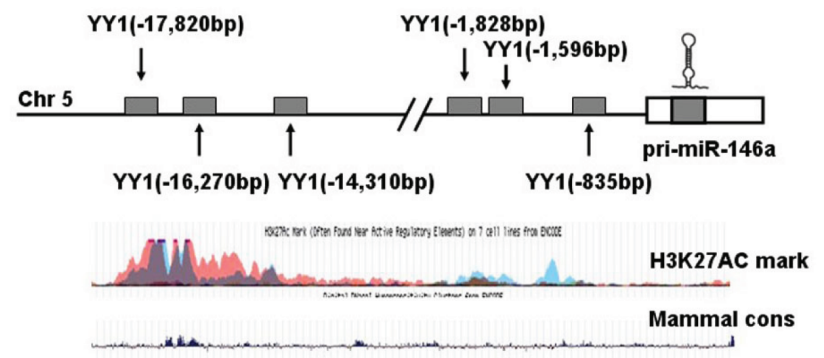

Fig. 1: The diagram illustrates the position of Yin Yang 1 (YY1) binding sites in miR-146a promoter.

Yin Yang 1 was discovered as a transcription factor and its transcriptional activity can be a repressor or an activator in different situations. As a ubiquitously expressed transcription factor, YY1 has attracted the interest of researchers not only because of its essential role in normal cell growth, but also due to its aberrant expression and potential regulatory function in different cancers. Yin Yang 1 has been reported to physically interact with a number of proteins regulating cell proliferation and apoptosis, such as p53, Mdm2, Ezh2, Rb, caspases and HDACs (13), indicating that YY1 works not only as a transcriptional factor but also as a critical joint of epigenetic modification. Therefore, as a multifunctional mediator of different signalling pathways, YY1 potentially acts as a critical regulator in cancer development and progression and likely plays a proliferative or oncogenic role in these processes.

Yin Yang 1 overexpression has been detected in prostate cancer, and YY1 expression was found to be correlated with malignant histological phenotypes (14). In mechanism research, YY1 inhibited prostate cancer Fas-induced and TRAIL-induced apoptosis through suppression of Fas and the death receptor 5 [DR5] (14). Moreover, YY1 is shown to be a coactivator of androgen receptor in promoting prostatespecific antigen (PSA) transcription, especially in androgendeprived circumstances, which indicates that YY1 potentially 
regulates prostate cancer development and progression through stimulating androgen receptor function. The aberrantly activated androgen receptor-signalling pathway in prostate cancer directly leads to CRPC transformation, which confirms that YY1 is associated with prostate cancer progression. The expression of YY1 has been reported to be controlled by a series of miRNAs like miR-7 (16), miR-29 (17), miR-34a (18) et al. Of interest, YY1 is also predicted to be the direct target of miR-146a in a bioinformatic miRNA target software [Fig. 2] (10), which is partly confirmed by the inverse expressions of miR-146a and YY1 in prostate cancer $(5,6)$.

\section{C. hsa-miR-146a/YY1 Alignment}

3. uugggUACCUUAAGUCAAGAGu $5^{\prime}$ hsa-miR-146a

773:5. aagaGUAGAGGU-AGUUCUCa $3^{\circ}$ YY1

Fig. 2: Predicted target site of miR-146a in the 3'-untranslated region (3'UTR) of Yin Yang 1 (YY1).

\section{CONCLUSION}

We present strong evidence indicating that both YY1 and miR-146a are involved in prostate cancer formation and progression though several means. Yin Yang 1 is overexpressed and miR-146a is found down-regulated in prostate cancer. Moreover, in bioinformatic analyses, YY1 is not only a direct target of miR-146a but also binds to the promoter region of miR-146a, which confirms the hypotheses that YY1-miR-146a-YY1 regulatory circuitry contributes to prostate cancer and may serve as a future intervention target.

\section{ACKNOWLEDGEMENTS}

This research was supported by National Natural Science Foundation of China (No. 81370849, 81300472, 81070592 and 81202034), Natural Science Foundation of Jiangsu Province (BL2013032 and BK2012336), Nanjing City (201201053), Southeast University (3290002402) and Science Foundation of Ministry of Education of China (20120092120071). The funders had no role in the study design, data collection and analysis, decision to publish, or preparation of the manuscript. The authors declare that they have no conflict of interest related to the publication of this manuscript.

\section{REFERENCES}

1. Siegel R, Naishadham D, Jemal A. Cancer statistics, 2013. CA Cancer J Clin 2013; 63: 11-30. doi: 10.3322/caac.21166. Epub 2013 Jan 17.

2. Feldman BJ, Feldman D. The development of androgen-independent prostate cancer. Nat Rev Cancer 2001; 1: 34-45.

3. Xie X, Lu J, Kulbokas EJ, Golub TR, Mootha V, Lindblad-Toh K et al Systematic discovery of regulatory motifs in human promoters and 3 UTRs by comparison of several mammals. Nature 2005; 434: 338-45.
4. Porkka KP, Pfeiffer MJ, Waltering KK, Vessella RL, Tammela TL, Visakorpi T. MicroRNA expression profiling in prostate cancer. Cancer Res 2007; 67: 6130-5.

5. Lin SL, Chiang A, Chang D, Ying SY. Loss of mir-146a function in hormone-refractory prostate cancer. RNA 2008; 14: 417-24. doi: 10.1261/rna.874808. Epub 2008 Jan 3.

6. Xu B, Wang N, Wang X, Tong N, Shao N, Tao J et al. MiR-146a suppresses tumor growth and progression by targeting EGFR pathway and in a p-ERK-dependent manner in castration-resistant prostate cancer. Prostate 2012; 72: 1171-8. doi: 10.1002/pros.22466. Epub 2011 Dec 7.

7. Deng Z, Wan M, Cao P, Rao A, Cramer SD, Sui G. Yin Yang 1 regulates the transcriptional activity of androgen receptor. Oncogene 2009; 28: 3746-57. doi: 10.1038/onc.2009.231. Epub 2009 Aug 10.

8. Tang S, Mishra M, Frazier DP, Moore ML, Inoue K, Deora R et al. Positive and negative regulation of prostate stem cell antigen expression by Yin Yang 1 in prostate epithelial cell lines. PLoS One 2012; 7: e35570. doi: 10.1371/journal.pone.0035570. Epub 2012 Apr 19.

9. Heinemeyer T, Wingender E, Reuter I, Hermjakob H, Kel AE, Kel OV et al. Databases on transcriptional regulation: TRANSFAC, TRRD and COMPEL. Nucleic Acids Res 1998; 26: 362-7.

10. Betel D, Koppal A, Agius P, Sander C, Leslie C. Comprehensive modeling of microRNA targets predicts functional non-conserved and non-canonical sites. Genome Biol 2010; 11: R90.

11. Galardi S, Mercatelli N, Farace MG, Ciafre SA. NF-kB and c-Jun induce the expression of the oncogenic miR-221 and miR-222 in prostate carcinoma and glioblastoma cells. Nucleic Acids Res 2011; 39: 3892-3902. doi: 10.1093/nar/gkr006. Epub 2011 Jan 18.

12. Schiffgen M, Schmidt DH, von Rucker A, Muller SC, Ellinger J. Epigenetic regulation of microRNA expression in renal cell carcinoma. Biochem Biophys Res Commun 2013; 436: 79-84. doi: 10.1016/ j.bbrc.2013.05.061. Epub 2013 May 22.

13. Nicholson S, Whitehouse H, Naidoo K, Byers RJ. Yin Yang 1 in human cancer. Crit Rev Oncog 2011; 16: 245-60.

14. Zaravinos A, Spandidos DA. Yin yang 1 expression in human tumors. Cell Cycle 2010; 9: 512-22.

15. Deng Z, Cao P, Wan MM, Sui G. Yin Yang 1: a multifaceted protein beyond a transcription factor. Transcription 2010; 1: 81-4.

16. Zhang $\mathrm{N}, \mathrm{Li} \mathrm{X}, \mathrm{Wu} \mathrm{CW}$, Dong $\mathrm{Y}$, Cai $\mathrm{M}$, Mok MT et al MicroRNA-7 is a novel inhibitor of YY1 contributing to colorectal tumorigenesis. Oncogene 2013; 32: 5078-88. doi: 10.1038/ onc.2012.526. Epub 2012 Dec 3.

17. Zhou L, Wang L, Lu L, Jiang P, Sun H, Wang H. A novel target of microRNA-29, Ring1 and YY1-binding protein (Rybp), negatively regulates skeletal myogenesis. J Biol Chem 2012; 287: 25255-65. doi: 10.1074/jbc.M112.357053. Epub 2012 May 31.

18. Chen QR, Yu LR, Tsang P, Wei JS, Song YK, Cheuk A et al. Systematic proteome analysis identifies transcription factor YY1 as a direct target of miR-34a. J Proteome Res 2011; 10: 479-87. doi: 10.1021/ pr1006697. Epub 2010 Dec 23.

Submitted 15 Nov 2013

Accepted 20 Jan 2014

Published 16 Jun 2014

Online: http://myspot.mona.uwi.edu/wimjopen/article/69

(c) Chen et al 2014

This is an open access article made freely available under Creative Commons Attribution 4.0 International (CC BY 4.0). Users are free to share, copy and adapt this work as long as the copyright holder (author) is appropriately and correctly credited. See http://creativecommons. org/licences/by/4.0/deed.en_us for more information. 